\title{
A Turn-off Fluorescent Sensor for Simple Detection of Vanillin Based on N-doped Carbon Dots
}

\author{
Hongyan Zeng ${ }^{1}$, Li Li1, Yaping Ding', Qi Zhuang ${ }^{1}$, Chaolin Wang ${ }^{1}$, Xiaojing Si ${ }^{2}$ \\ ${ }^{1}$ Department of Chemistry, Shanghai University, Shangda Road, Baoshan District, Shanghai 200444, \\ P.R.C \\ ${ }^{2}$ School of Public Health, Shanghai Aurora College, Shanghai 201908, PR China \\ Corresponding e-mail address: wdingyp@sina.com, lilidu@shu.edu.cn
}

\begin{abstract}
:
Vanillin (Van) is an important fragrance, which is widely used as flavoring agents or perfume fixative in food, cosmetics, tobacco industry, medicine, etc. To our knowledge, carbon dots were first applied as fluorescence sensor for the quantitative determination of Van. The as-synthesized N-CDs were characterized by transmission electron microscopy (TEM), the fourier transform infrared spectra (FT-IR), UV spectrophotometer and spectrofluorophotometer, respectively. The fluorescent detection of Van was successfully conducted in aqueous media and incubation time was as short as $5 \mathrm{~min}$. The proposed probe showed a great linear relationship between fluorescence intensity function $\log \left(\mathrm{F}_{0} / \mathrm{F}\right)$ and concentration of Van in range from $5 \mu \mathrm{M}$ to $140 \mu \mathrm{M}$ with a detection limit $(\mathrm{S} / \mathrm{N}=3)$ of $0.4 \mu \mathrm{M}$.
\end{abstract}

Key words: carbon dots, Vanillin, fluorescent sensor, simple detection, turn-off

\section{Introduction}

Vanillin (4-hydroxy-3-methoxybenzaldehyde, Van) is an important flavouring extracted from vanilla beans, also is one of the largest productive spices in synthetic fragrances over the world. It is widely used as flavoring agents or perfume fixative in food [1], cosmetics, tobacco industry, medicine, etc. However, it can still be harmful to human's health when the concentration of Van is high enough [2]. Therefore, the determination of Van in various products is required. Comparing to other detecting method, fluorescent sensor is an alternative detection method which has some superior advantages of simple operation, high sensitivity and cost-effectiveness for the trace analysis ${ }^{[3]}$. Carbon dots have been widely used as good fluorescent probes in the detection of various substances ${ }^{[4]}$ due to their great water solubility, photochemical stability and strong fluorescence.

In this work, we use p-Phthalic acid and ethylenediamine as carbon source and nitrogen source, respectively. Then the $\mathrm{N}-\mathrm{CD}$ s were synthesized by a simple one-pot hydrothermal method. The as-prepared $\mathrm{N}-\mathrm{CDs}$ were first used as the fluorescent probe for the detection of Van.

\section{Characterization of $\mathrm{N}-\mathrm{CDs}$}

Fig. 1 is the morphology of the as-synthesized $\mathrm{N}-\mathrm{CD}$ investigated by TEM. As shown in Fig. 1, the $\mathrm{N}-\mathrm{CDs}$ are uniform spherical nanoparticles and the diameter range from 3 to $5 \mathrm{~nm}$.

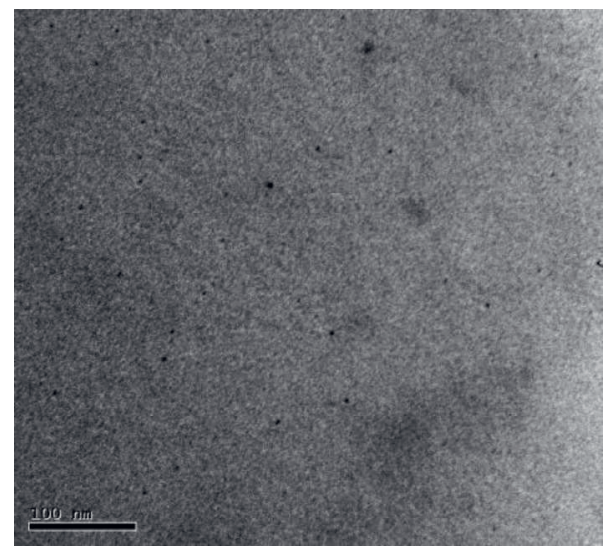

Fig. 1 TEM of $N-C D S$

As depicted in Fig. 2A, most spectral peaks of $\mathrm{N}-\mathrm{CDs}$ are similar to that of PTA, except for the strong peaks located at 1544 and $1369 \mathrm{~cm}^{-1}$ which are attributed to bending vibration of $\mathrm{N}-\mathrm{H}$ and stretching vibration of $\mathrm{C}-\mathrm{N}$, respectively. And the new peak of N-CDs located at 3327 $\mathrm{cm}^{-1}$ is attributed to stretching vibration of $\mathrm{N}-\mathrm{H}$. The above descriptions indicated that nitrogen is successfully doped in the carbon dots. In 
order to explore the optical properties of the $\mathrm{N}$ CDs, UV-Vis absorption and fluorescence spectra are obtained in Fig. 2B, the N-CDs typically display optical absorption in the UV-Vis region with an absorption peak at $240 \mathrm{~nm}$, and a strong emission peak at $430 \mathrm{~nm}$ appeared upon excitation at $350 \mathrm{~nm}$. The excitation and emission spectra of carbon dots are symmetrical.

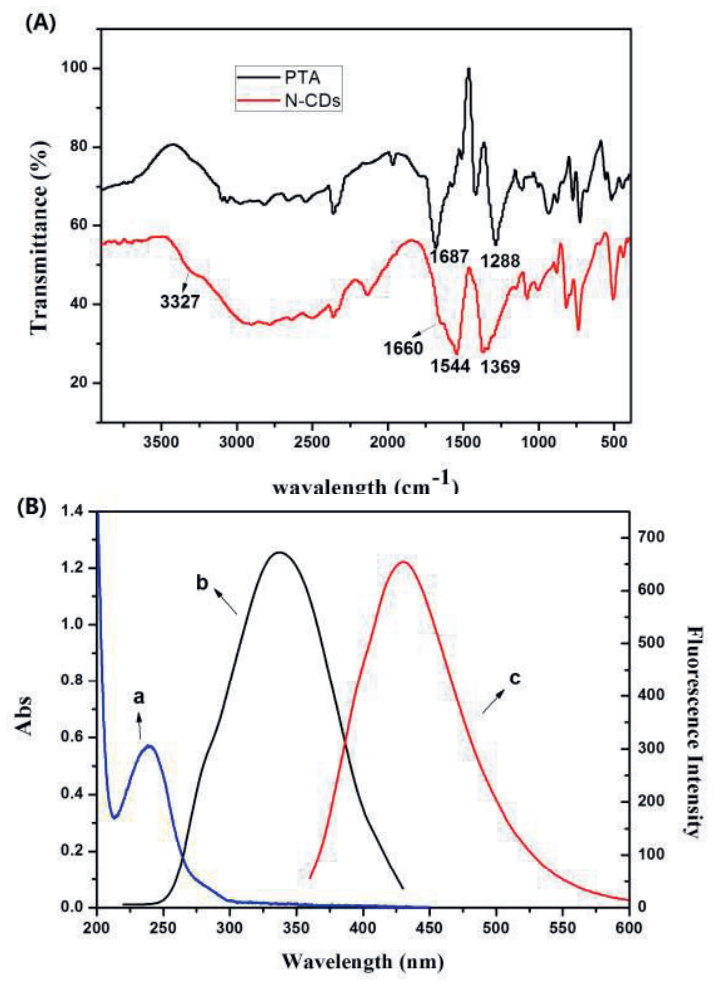

Fig. 2 (A) FT-IR spectra of p-Phthalic acid(PTA) and $N-C D s ;(B)$ a) UV-Vis absorption, b) fluorescence excitation and c) fluorescence emission spectra of $\mathrm{N}$ CDs.

\section{Experimental result}

In order to explore the optimal detecting environment of fluorescent sensor, the optimization experiments of $\mathrm{pH}$ and reaction time was carried out in this work. As depicted in Fig. $3 A$ and Fig. 3B, we chose water as the reaction media and 5 minutes as reaction time for our experiment, which made the sensor more simple and time-saving. As Fig.3C shows that the fluorescence intensity of N-CDs decreased obviously with gradually increased concentration of Van from $5 \mu \mathrm{M}$ to $140 \mu \mathrm{M}$. And Fig.3D demonstrates that the fluorescence intensity function $\log \left(F_{0} / F\right)$ are linearly increase with Van's concentrations. The corresponding linear regression equation was $\log \left(\mathrm{F}_{0} / \mathrm{F}\right)=$ $0.0116 C_{V a n}+0.0149(R=0.999)$, where $F_{0}$ and $\mathrm{F}$ are the fluorescence intensities of $\mathrm{N}-\mathrm{CDs}$ at $350 \mathrm{~nm}$ in the absence and presence of Van, respectively. The detection limit was calculated as $0.4 \mu \mathrm{M},(\mathrm{S} / \mathrm{N}=3)$.
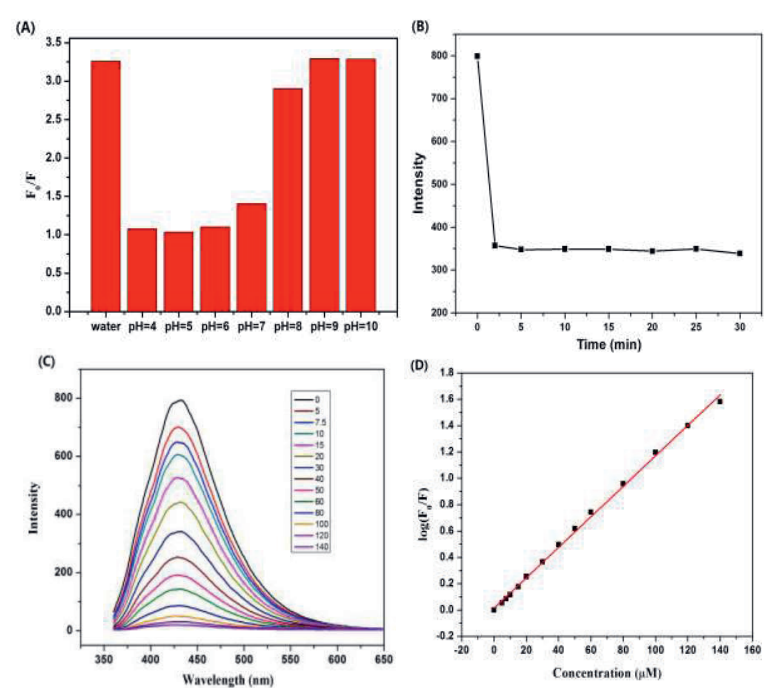

Fig.3 (A) The relative fluorescence intensity ( $\left.F_{0} / F\right)$ at different $\mathrm{pHs}$ and water; (B) Effect of incubation time on the fluorescence intensity of (N-CDs)-Van system; (C) Fluorescence spectra of $\mathrm{N}-\mathrm{CDs}$ with addition of different concentrations of Van from 0 to $140 \mu M$; (D) The linear relationship between $\log \left(F_{0} / F\right)$ and Van concentration in the range of 0-140 $\mu \mathrm{M}$, where $F_{0}$ and $F$ are fluorescence intensities in the absence and presence of Van, respectively.

This work is supported by the National Natural Science Foundation of China (No. 21671132)

\section{References}

[1] F. Bettazzi, I. Palchetti, S. Sisalli, M. Mascini, A Disposable Electrochemicalsensor for Vanillin Detection, Anaytical Chimica Acta 555,134-138 (2006); doi: 10.1016/j.aca.2005.08.069

[2] P.H. Deng, Z.F. Xu, R.Y. Zeng, C.X. Ding, Electrochemical Behavior and Voltammetric Determination of Vanillin Based on An Acetylene Black Pasteelectrode Modified with GraphenePolyvinylpyrrolidone Composite Film, Food Chemistry 180, 156-163 (2015); doi: 10.1016/j.foodchem.2015.02.035

[3] J. Hou, J. Dong, H. Zhu, X. Teng, S. Ai, M. Mang, A Simple and Sensitive Fluorescent Sensor for Methyl Parathion Based on L-tyrosine Methyl Ester Functionalized Carbon Dots, Biosensors \& Bioelectronics 68, 20-26 (2015); doi: 10.1016/j.bios.2014.12.037

[4] L. Liu, F. Feng, M.C. Paau, Q. Hu, Y. Liu, Z. Chen, Y. Bai, F. Guo, M.M.F. Choi, Sensitive Determination of Kaempferol Using Carbon Dots as A Fluorescence Probe, Talanta 144, 390-397 (2015); doi: 10.1016/j.talanta.2015.07.004 